\title{
КОММЕНТАРИИ. ДИСКУССИЯ. КРИТИКА
}

\section{Замечание к статье П. Е. Рябова \\ «Явное интегрирование \\ и топология случая Д. Н. Горячева»}

\section{А. В. Цыганов}

Рассмотрим интегрируемую систему с интегралами движения

$$
\begin{gathered}
H=\frac{1}{2}\left(M_{1}^{2}+M_{2}^{2}\right)+M_{3}^{2}+\frac{1}{2} c\left(\alpha_{1}^{2}-\alpha_{2}^{2}\right)+\frac{b}{\alpha_{3}^{2}}, \quad b, c \in \mathbf{R}, \\
K=\left(M_{1}^{2}+M_{2}^{2}+\frac{b}{\alpha_{3}^{2}}\right)^{2}+2 c \alpha_{3}^{2}\left(M_{1}^{2}-M_{2}^{2}\right)+c^{2} \alpha_{3}^{4},
\end{gathered}
$$

используя обозначения, принятые в обсуждаемой статье [4]. Скобки Пуассона между динамическими переменными

$$
\left\{M_{i}, M_{j}\right\}=\varepsilon_{i j k} M_{k}, \quad\left\{M_{i}, \alpha_{j}\right\}=\varepsilon_{i j k} \alpha_{k}, \quad\left\{\alpha_{i}, \alpha_{j}\right\}=0,
$$

являются скобками Ли-Пуассона на алгебре Ли $e^{*}(3)$. Так как

$$
\{H, K\}=8 c \alpha_{3} M_{1} M_{2}\left(\alpha_{1} M_{1}+\alpha_{2} M_{2}+\alpha_{3} M_{3}\right),
$$

то данная система является интегрируемой только при нулевом значении интеграла площадей

$$
C=\alpha_{1} M_{1}+\alpha_{2} M_{2}+\alpha_{3} M_{3}=0 .
$$

При $b=0$ эта интегрируемая система и переменные разделения для нее были построены C. А. Чаплыгиным в работе [5]. Обобщение результатов Чаплыгина на случай пучка скобок Пуассона приведено в книге [2].

Сингулярное слагаемое к гамильтониану было добавлено Д. Н. Горячевым в работе [3]. Доказательство того, что переменные разделения Чаплыгина являются переменными разделения и для случая Горячева $b \neq 0$, приведено в работе [9].

Получено 30 сентября 2011 года

Цыганов Андрей Владимирович andrey.tsiganov@gmail.com Санкт-Петербургский государственный университет 199034, Россия, г. Санкт-Петербург, Университетская наб., 7-9 
Случаи Чаплыгина и Горячева являются частными случаями так называемого гиростата Ковалевской - Горячева - Чаплыгина, гамильтониан которого отличается от приведенного выше (1) добавлением линейных по моментам $M$ и по координатам $\alpha$ слагаемых. Комплексные переменные для данной более общей системы приведены в [8]. Обсуждение комплексных и вещественных переменных разделения для данной конкретной системы, соответствующих $r$-матриц и широкого круга вопросов, связанного с применимостью метода разделения переменных для других интегрируемых систем, может быть найдено в книге [1].

В работе [4] утверждается, что новые переменные разделения для случая Горячева могут быть найдены с помощью геометрического метода Харламова. При $c=1$ и $\alpha_{1}^{2}+$ $+\alpha_{2}^{2}+\alpha_{3}^{2}=1$ по определению (см. формулу (7) в [4]) данные переменные $u_{1}, u_{2}$ являются корнями полинома

$$
z u^{2}-2 b u+(2 b \xi-k z)=0, \quad \text { где } \quad z=\alpha_{3}^{2}, \quad \xi=M_{1}^{2}+M_{2}^{2}+\frac{b}{\alpha_{3}^{2}}
$$

и $k$ - постоянная интеграла $K$. Однако легко проверить, что корни данного полинома не коммутируют друг с другом,

$$
\left\{u_{1}, u_{2}\right\} \neq 0 \text {, }
$$

относительно исходной скобки Пуассона (2) даже при нулевом значении интеграла площадей (3) и, тем самым, не являются переменными разделения. Напомним, что, согласно [6], переменными разделения называются канонические относительно скобки Пуассона переменные.

Таким образом, применение геометрического метода Харламова к случаю Горячева в работе [4] приводит к некоммутирующим относительно скобки Пуассона переменным, которые не являются переменными разделения. Для того чтобы понять, что же дает метод Харламова в обсуждаемом случае, мы перейдем к переменным разделения Чаплыгина [5]:

$$
q_{1,2}=\frac{M_{1}^{2}+M_{2}^{2} \pm h}{\alpha_{3}^{2}}, \quad h^{2}=\left(M_{1}^{2}-M_{2}^{2}+\alpha_{3}^{2}\right)^{2}+4 M_{1}^{2} M_{2}^{2} .
$$

На нулевом уровне интеграла площадей переменные $q_{1,2}$ коммутируют друг с другом,

$$
\left\{q_{1}, q_{2}\right\}=0
$$

относительно исходной скобки Пуассона, как и положено в методе разделения переменных [6]. Используя эти переменные $q_{1,2}$ и соответствующие канонически сопряженные импульсы $p_{1,2}[9,10]$, некоммутирующие корни полинома (4) можно переписать в следующем виде:

$$
u_{1}=8\left(q_{1}^{2}-1\right) p_{1}^{2}-2 q_{1}+2 H, \quad u_{2}=8\left(q_{2}^{2}-1\right) p_{2}^{2}-2 q_{2}+2 H .
$$

Так как любая функция от пары сопряженных переменных разделения вида $s_{i}\left(p_{i}, q_{i}\right)$ также является переменной разделения [6], то можно определить следующий набор коммутирующих переменных разделения:

$$
s_{1}\left(q_{1}, p_{1}\right)=u_{1}-2 H, \quad s_{2}\left(q_{2}, p_{2}\right)=u_{2}-2 H .
$$

Заметим, что абсолютно такой же сдвиг вспомогательных переменных $u_{1,2}$ для получения коммутирующих переменных разделения $s_{1,2}$ использовала и С. В. Ковалевская [7] в своем случае. 
Таким образом, становится понятно, что для построения настоящих переменных разделения в случае Горячева геометрический метода Харламова приводит к ошибочным результатам и, следовательно, его необходимо дополнить (например, методами пуассоновой геометрии или другими методами). Только в этом случае мы действительно получим переменные разделения, которые, тем не менее, полностью эквивалентны известным переменным разделения Чаплыгина.

\section{Список литературы}

[1] Борисов А. В., Мамаев И. С. Современные методы теории интегрируемых систем. М.-Ижевск: ИКИ, 2003. $296 \mathrm{c}$.

[2] Борисов А. В., Мамаев И. С. Динамика твердого тела: гамильтоновы методы, интегрируемость, хаос. М.-Ижевск: ИКИ, 2005. 576 с.

[3] Горячев Д.Н. Новые случаи интегрируемости динамических уравнений Эйлера // Изв. Варшавского ун-та, 1916, т. 3, с. 1-13.

[4] Рябов П.Е. Явное интегрирование и топология случая Д.Н. Горячева // Докл. РАН, 2011, т. 439 , вып. 3, с. 315-318.

[5] Чаплыгин С. А. Новое частное решение задачи о движении твердого тела в жидкости // Тр. отд. физ. наук общ-ва любителей естествознания, 1903, т. 11, вып. 2, с. 7-10.

[6] Jacobi C. G. J. Vorlesungen über Dynamik. Berlin: G. Reimer, 1884. 300 pp.

[7] Kowalevski S. Sur le probléme de la rotation d'un corps solide autour d'un point fixe // Acta Math., 1889, vol. 12, pp. 177-232.

[8] Tsiganov A. V. On the Kowalevski-Goryachev-Chaplygin gyrostat // J. Phys. A, 2002, vol. 35, no. 26, L309-L318.

[9] Tsiganov A. V. On the generalized Chaplygin system // Вопросы квантовой теории поля и статистической физики. Т. 21, Зап. научн. сем. ПОМИ, 374, ПОМИ, СПб., 2010, с. 250-267 [J. Math. Sci. (N. Y.), 2010, vol. 168, no. 8, pp. 901-911.

[10] Tsiganov A. V., Khudobakhshov V. A. Integrable systems on the sphere associated with genus three algebraic curves // Regul. Chaotic Dyn., 2011, vol.16, nos. 3-4, pp. 396-414.

\section{Comments on P. E. Ryabov «Explicit integration and topology of D. N. Goryachev case»}

Andrey V. Tsiganov

Saint-Petersburg State University

Universitetskaya nab. 7-9, St. Petersburg, 199034, Russia

andrey.tsiganov@gmail.com

Received September 30, 2011

Citation: Rus. J. Nonlin. Dyn., 2011, vol. 7, no. 3, pp. 715-717 (Russian) 